\title{
Modified Traffic Flow Model with Connected Vehicle Microscopic Data for Proactive Variable Speed Limit Control
}

\author{
Jie Fang $\left(\mathbb{D},{ }^{1}\right.$ Huixuan Ye, ${ }^{1}$ and Said M. Easa $\mathbb{D}^{2}$ \\ ${ }^{1}$ Transportation Department, Fuzhou University, Fuzhou, Fujian, China \\ ${ }^{2}$ Department of Civil Engineering, Ryerson University, Toronto, Ontario, Canada \\ Correspondence should be addressed to Jie Fang; fangjie@fzu.edu.cn
}

Received 16 February 2019; Revised 16 April 2019; Accepted 22 May 2019; Published 10 June 2019

Guest Editor: Md. A. S. Kamal

Copyright (c) 2019 Jie Fang et al. This is an open access article distributed under the Creative Commons Attribution License, which permits unrestricted use, distribution, and reproduction in any medium, provided the original work is properly cited.

\begin{abstract}
Most previous prediction based Variable Speed Limit (VSL) control strategies focused on improving traffic mobility based on the macroscopic traffic data. Nowadays, the emerging technologies provide access to the microscopic traffic flow data, which better captures the details of traffic flow dynamics in the VSL controlled environment. Thus, in this paper, the microscopic traffic flow data were utilized as a supplement to predict the evolutions of traffic flow parameters. The proposed VSL control algorithm adopts the Model Predictive Control (MPC) framework, which employs a modified version of the classic traffic flow model METANET to take advantage of the microscopic data in traffic flow predictions. The microscopic traffic simulation software VISSIM was used to establish an experimental simulation platform and perform real time traffic responsive control based on field data. The proposed control strategy was evaluated against the no-VSL control and macroscopic-based VSL controlled scenario. The results show that utilizing the proposed modified METANET model reduced the error in speed prediction accuracy and improved system mobility performance.
\end{abstract}

\section{Introduction}

Urban freeways provide efficient and convenient traffic service for road users and play a significant role in accelerating the development of regional economy [1]. With the rapid economic development, the great increase in car-ownership has aggravated the traffic congestion. Thus, traffic mobility and safety have become major challenges in freeway operations. Due to the restriction of urban space and the high cost of infrastructure construction, the problems may not be solved simply by expanding road facilities. To ease traffic congestion, some traffic control measures have been adopted, such as Route Guidance [2, 3], Ramp Metering [4, 5], and Variable Speed Limits (VSL). Among these measures, VSL control draws widely attentions. It determines a dynamic speed limit according to the current traffic flow states, climatic, road environments, etc. The main benefits of VSL control are as follows: (1) improves traffic operations through regulating the mainstream traffic flow and delaying the forthcoming traffic breakdown for potential capacity gain and higher level of service, (2) mitigates the speed differences between individual vehicle for fewer traffic collisions, and (3) reduces the vehicle stop frequencies for vehicle emission and air pollution [6-8].

The MPC control framework has recently been widely adopted in proactive freeway control simulations implementing VSL. The essential core of the MPC framework is the included traffic state prediction model for process control. For this approach, the performance of VSL control strategy depends heavily on the accurate prediction of traffic-flow parameters, which will be used for quantitatively determining the dynamic speed limits. To adapt the limitation of the traditional data collection methods, existing VSL control methods adopt macroscopic traffic flow data that can be easily collected, such as speed, volume, and density. Enabled by the fast-emerging technologies, such as internet of vehicles, the real-time microscopic traffic data, such as the acceleration rate, can be collected by the in-vehicle or roadside sensors [9]. This newly available data source may better capture the details of traffic flow dynamics in the VSL controlled environment. 
Thus, in this paper, a VSL control strategy based on a modified METANET model utilizing the microscopic traffic data was proposed. In the modified model, the microscopic data are used in the prediction model as a supplement to predict the evolution of the traffic dynamics. By analyzing higher resolution data, such as the individual acceleration rate and headway at second-by-second level, the responding and evolving of the traffic flow to the control measure can be modeled more accurately, thus improves the prediction accuracy. The modified model with proposed control strategy was implemented in a simulated freeway to validate their feasibility and control performance.

The next sections present a brief literature review of existing VSL control strategies, followed by the descriptions of the basic and modified METANET models. The following sections present the model validation through an application of the proposed control strategy using VISSIM simulation and the conclusions.

\section{Literature Review}

The studies of VSL control in Europe and North America, traced back to 1990s, have provided valuable state-of-the-art and practical experiences [10]. Various VSL control strategies have been proposed and can be grouped into three categories: rule-based VSL control strategies, feedback controller-based VSL control strategies, and model-based VSL control strategies.

The rule-based control strategies use real-time traffic measurements as a basis for real-time control. The decision tree strategy can be categorized as rule-based control strategy, which is the earliest to be developed for VSL control. It uses an algorithm that defines an indicator, such as density, as a criterion for determining whether to start the VSL control. Decision tree-based strategies are straightforward for field implementation. Lee et al. [11] proposed a real-time conflict prediction model and showed that the precursors of conflict could be decided in an objective manner to replace the subjective classification mode used in the analysis. In 2006, Lee et al. [12] proposed a VSL strategy that reduced the speed limit when the potential conflict exceeded a preset threshold. The optimal speed limit was selected based on several thresholds associated with safety. The results suggested that the number of potential conflicts were decreased, but travel time was increased.

In 2011, Carlson et al. [13] designed a simple local feedback controller Mainstream Traffic Flow Control (MTFC) to improve traffic-flow efficiency. The control strategy was evaluated using simulation and the performance of the controller was shown to approach the optimal control effect. In 2013, the same group of authors [14] proposed two simple feedback controllers that relied on readily available real-time measurements for local MTFC via VSL. The results showed that the feedback controllers had satisfactory control effects. Recently, Iordanidou et al. [15, 16] proposed an extended feedback-based VSL control strategy, considering multiplebottleneck locations, and obtained good results.

The Model Predictive Control- (MPC-) based VSL control strategy is a model-based VSL control strategy, of which the model has predictive function. The limitation of the rule-based control strategies is that traffic conditions may have already broken down before VSL is deployed. Thus, Model Predictive Control (MPC) has emerged as a new approach to address this limitation. The MPC is a valuable, widely used framework for VSL control of freeways [17]. In model experiment, future traffic conditions (e.g., congestion) are foreseen before they even occur, and VSL strategies are deployed to reduce traffic volume in the expected congestion area, see, for example, Khondaker et al. [18]. The framework uses a model to predict future traffic states. The MPC-based VSL control strategies consider future traffic conditions and quantitatively evaluate the impact of VSL control on trafficflow dynamics to keep traffic flow at high efficiency, especially during congested periods. In 2005, Zhang et al. [19] used MPC to design a roadway controller that reduced traffic congestion. In 2009, Zegeye et al. [20] used MPC to evaluate the impact of dynamic speed limit control and the results showed a reduction in total time spent. In 2010, Ghods et al. [21] solved the problem of real-time optimal control of traffic flow in a freeway network with a promising approach by casting the underlying dynamic control problem in an MPC framework. Hadiuzzaman et al. proposed a modified Cell Transmission Model (CTM) based on Daganzo's original model [22] and used MPC to alter the speed limit dynamically [23, 24]. In 2017, Han et al. [25] developed a fast MPC based approach for VSL coordination to resolve freeway jam waves. This MPC approach is based on a more accurate discrete first-order model that keeps the linearity property of the classical discrete first-order model and takes capacity drop into consideration. The simulation results demonstrated that the proposed control strategy resolved the jam wave with a real-time feasible computation speed [26].

As a macroscopic modeling tool, the METANET model, developed by Papageorgiou et al. [10], and its extensions are widely used. One of the pioneering MPC-based VSL control strategies was proposed by Hegyi [27] and Hegyi et al. $[28,29]$. The authors modified the METANET model, for example, by proposing a revised the desired speed term. The new desired speed is the minimum of the targeted speed based on the current traffic conditions and the displayed speed limit. The MPC framework was adopted to determine the optimal speed limit. Hence, Hegyi et al. [29] proposed an extended METANET model with modeling of dynamic speed limits and mainstream origins. The results showed that the VSL can prevent traffic breakdown and maintain a higher outflow. In 2010, Carlsonet al. [30] incorporated VSL in the METANET model as an additional control component leading to an extended optimal control formulation. The results showed that traffic-flow efficiency was substantially improved when VSL control measures were used. In 2012, Hadiuzzaman et al. [23] replaced the fundamental diagram with the VSL control variable in the relaxation term of the METANET model, the proposed traffic dynamics with the control strategy were implemented in a freeway corridor using the MPC framework. The analysis was carried out in VISSIM and the results showed that VSL was mostly effective during congestion periods in terms of mobility. In 2014, Sun et al. [31] proposed a new extension of METANET model in 
which traffic state variables were modeled and predicted. In addition, VSL values were optimized using MPC. Yu et al. [32] proposed an extended METANET model, in which the desired speed term was modified to minimize the total crash risk. The results showed that traffic safety improved and speed homogeneity was enhanced. The MPC based VSL control have been proven effective in the preceding researches.

However, most previous VSL control strategies predicted traffic-flow states using collected macroscopic traffic data to determine the VSL control signal. Due to lack of microscopic traffic flow data, the prediction accuracy maybe compromised in certain circumstances, such as low density (free flow). With the development of new sensoring and communication technologies, microscopic traffic data can be collected and incorporated into the formulation of the basic METANET model for better modeling the responding and evolving of the traffic flow under VSL controlled environment. Thus, in this paper, a modified METNET model incorporating microscopic traffic data will be proposed to establish an MPC based proactive VSL control strategy that further improves the prediction accuracy and freeway operation efficiency.

\section{Methodology}

\subsection{Model Formulation}

3.1.1. Macroscopic METANET Model. In this paper the authors adopted the MPC framework that incorporates the METANET model and its extensions, which are valuable tools widely used to make accurate prediction of traffic-flow variables. The METANET model is deterministic, discretetime, discrete-space, and macroscopic, making it very suitable for model-based traffic control [27].

According to the conservation equation of fluid motion,

$$
\frac{\partial_{\rho}}{\partial_{t}}+\frac{\partial_{q}}{\partial_{x}}=H^{\prime}-S^{\prime}
$$

where $H^{\prime}=$ average inflow from onramp at position $x$ (veh) and $S^{\prime}=$ average outflow of the off-ramp at position $x$ (veh).

If the number of lanes of segment $i$ is $\lambda_{i}$ and the length of segment $i$ is $L_{i}$, (1) becomes

$$
\begin{gathered}
\frac{\lambda_{i}\left[\rho_{i}(k+1)-\rho_{i}(k)\right]}{T}+\frac{q_{i}(k)-q_{i-1}(k)}{L_{i}} \\
=\frac{h_{i}(k)-s_{i}(k)}{L_{i}}
\end{gathered}
$$

After adjustment, the conservation equation of vehicles is obtained as

$$
\begin{aligned}
\rho_{i}(k+1)= & \rho_{i}(k) \\
& +\frac{T}{L_{i} \lambda_{i}}\left(q_{i-1}(k)-q_{i}(k)+h_{i}(k)-s_{i}(k)\right)
\end{aligned}
$$

where $\rho_{i}(k)=$ density of segment $i$ at time $k(\mathrm{veh} / \mathrm{km} / \mathrm{ln})$; $q_{i}(k)=$ traffic flow of segment $i$ at time $k(\mathrm{veh} / \mathrm{h}) ; h_{i}(k)=$ onramp flow of segment $i$ at time $k(\mathrm{veh} / \mathrm{h})$ and $s_{i}(k)=$ off-ramp flow of segment $i$ at time $k$ (veh/h).

The outflow of segment $i$ is equal to the density multiplied by the average speed and the number of lanes of that segment. That is,

$$
q_{i}(k)=\rho_{i}(k) \cdot v_{i}(k) \cdot \lambda_{i}(k)
$$

When adjusting towards the desired speed, there will a brief delay related to the drivers' reaction time and vehicle acceleration capability. In other words, to reach the desired speed at position $\triangle x$ ahead, a certain time and spacing are required for the vehicle to adjust according to the observation of the downstream traffic-flow state. If the adjustment time is $\triangle t$, then

$$
v(x, t+\Delta t)=U[\rho(x+\Delta x, t)]
$$

Applying Taylor series expansion to each side of (5), then

$$
\begin{aligned}
& v(x, t)+\Delta t \cdot \frac{\partial v(x, t)}{\partial t} \\
& \quad=U[\rho(x, t)]+\Delta x \cdot \frac{d U[\rho]}{d \rho} \cdot \frac{\partial \rho(x, t)}{\partial x}
\end{aligned}
$$

In previous researches, $\triangle x$ was set to be an average value of $1 /(2 \rho)$ based on the empirical data $[10,27]$, while $(d U[\rho] / d \rho)$ is the sensitivity of adjusting towards the anticipated speed, considering segment density. Defining $(d U[\rho] / d \rho)=\gamma(x, t),(6)$ becomes

$$
\begin{aligned}
& v(x, t)+\Delta t \cdot \frac{\partial v(x, t)}{\partial t} \\
& =U[\rho(x, t)]+\frac{\gamma(x, t)}{2 \rho} \cdot \frac{\partial \rho(x, t)}{\partial x}
\end{aligned}
$$

where

$$
\frac{\partial v(x, t)}{\partial t}=\frac{\partial v}{\partial x} \cdot \frac{\mathrm{d} x}{\mathrm{~d} t}+\frac{\partial v}{\partial t}
$$

Based on (8), (7) can be written as

$$
\frac{\partial v}{\partial t}=\frac{1}{\Delta t}\left\{U[\rho]+\frac{\gamma}{2 \rho} \cdot \frac{\partial \rho}{\partial x}-v\right\}-v \cdot \frac{\partial v}{\partial x}
$$

After discretizing and rearranging (9), one obtains

$$
\begin{aligned}
v_{i}(k+1)= & v_{i}(k)+\frac{T}{\tau_{i(k)}}\left(U\left[\rho_{i}(k)\right]-v_{i}(k)\right) \\
& -\frac{T}{L_{i}} v_{i}(k)\left[v_{i}(k)-v_{i-1}(k)\right]+\frac{\gamma_{i(k)} T}{\tau_{i(k)} L_{i}} \\
& \cdot \frac{\rho_{i+1}(k)-\rho_{i}(k)}{\rho_{i}(k)+\kappa} \\
U\left[\rho_{i}(k)\right]= & v_{\text {free }, i} \cdot \exp \left[-\frac{1}{a_{m}}\left(\frac{\rho_{i}(k)}{\rho_{\text {crit }}}\right)^{a_{m}}\right]
\end{aligned}
$$




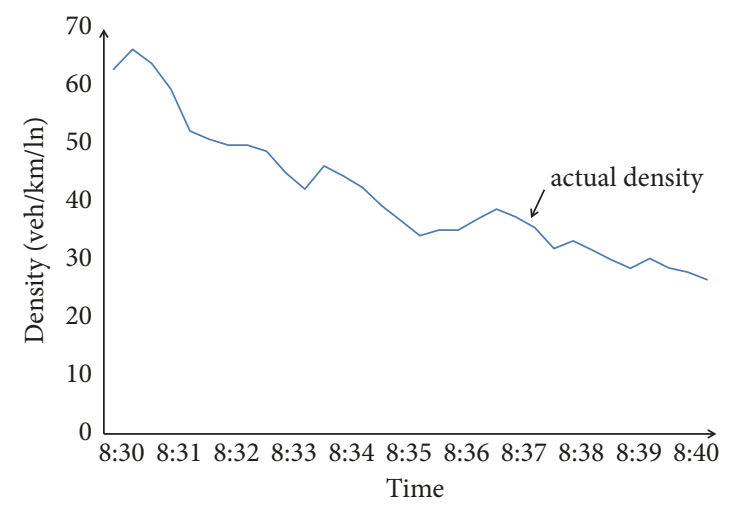

(a)

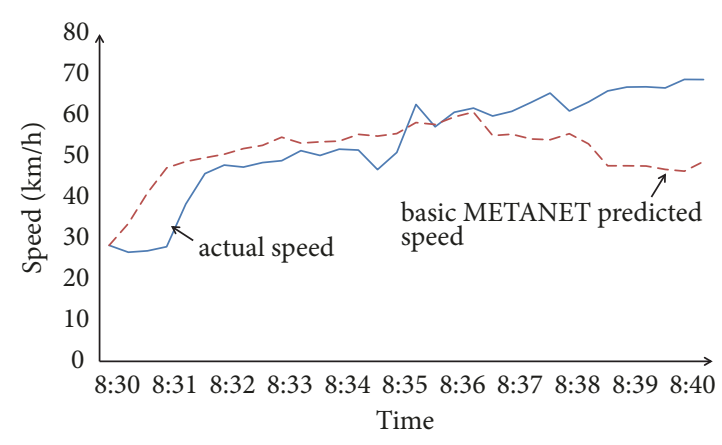

(b)

FIGURE 1: Variation of density corresponding actual and basic METANET predicted speeds: (a) actual density and (b) actual speed and basic METANET predictions.

where $\mathrm{U}\left[\rho_{\mathrm{i}}(\mathrm{k})\right]=$ desired speed of segment $i(\mathrm{~km} / \mathrm{h}), \gamma_{\mathrm{i}(\mathrm{k})}$ $=\mathrm{a}$ parameter with negative value, sensitivity of adjusting towards the anticipated speed of segment $i$ at time $k, \tau_{\mathrm{i}(\mathrm{k})}=$ driver adjustment delay coefficient of segment $i, \kappa=$ positive compensation coefficient to avoid the error brought by toosmall $\rho_{i}(k), \mathrm{v}_{\text {free, } \mathrm{i}}=$ free-flow speed of segment $i(\mathrm{~km} / \mathrm{h}), \mathrm{a}_{\mathrm{m}}$ $=$ model parameter of segment, and $\rho_{\text {crit }}=$ critical density (veh/km/ln).

In the basic METANET model, $\gamma_{\mathrm{i}(\mathrm{k})}, \tau_{\mathrm{i}(\mathrm{k})}$, and $\kappa$ are treated as constant model parameter. Thus, (10) becomes

$$
\begin{aligned}
v_{i}(k+1)= & v_{i}(k)+\frac{T}{\tau}\left(U\left[\rho_{i}(k)\right]-v_{i}(k)\right) \\
& -\frac{T}{L_{i}} v_{i}(k)\left[v_{i}(k)-v_{i-1}(k)\right]+\frac{\gamma T}{\tau L_{i}} \\
& . \frac{\rho_{i+1}(k)-\rho_{i}(k)}{\rho_{i}(k)+\kappa}
\end{aligned}
$$

3.1.2. Proposed Macroscopic METANET Model with Microscopic Connected Vehicle Data. The term $\triangle x$ in (6) indicates the lag of speed adjustments. In the basic METANET model, $\triangle x$ is assumed to an empirical averaged value of $1 /(2 \rho)$, and $(d U[\rho] / d \rho)$ was replaced as a constant system parameter. In other words, the distance required for speed adjustment was set to be half of the headway, by simply assuming averaged vehicle headway as the ideal situation. However, this assumption is not always consistent with field implementation. Although this assumption is relatively accurate under high density, under low density circumstances the large averaged headway will cause significant model mismatches, as illustrated in Figure 1.

As noted in Figure 1, before 8:35 am, the density is comparatively higher and the prediction error of the basic METANET model is lower than 35\%. On the other side, after 8:35 am, prediction error of the basic METANET model grows larger as the density decreases. Noticing this model mismatches, an extra model parameter $\kappa$ was introduced into the basic METANET model as compensation coefficient to reduce the error in the low-density region [10]. Nonetheless, the error of speed prediction at low density may still be large in some cases, as shown in Figure 1(b). Therefore, a model modification utilizing the microscopic data was proposed to overcome this mismatch in this paper.

In the basic METANET model, since the actual individual vehicle status is unknown, the vehicle is assumed to be equally distributed along the road segment (by taking the segment averaged headway). Thus, the distance required for speed adjustment $\Delta x$ was set to half of the headway, which clearly will not represent all the circumstances in the real world. This distance required for speed adjustment should be a function of the current traffic condition: influenced by the interactions between individual vehicles, or in the other words, individual vehicle spacing when the traffic is congested and affected by the individual driving characteristic (acceleration/deceleration status) when the traffic is light. Therefore, taking advances of the state-of-the-art technologies, microscopic data were collected in this study to derive a modified METANET model, which utilized the microscopic data to model this dynamic term. In the field, when the density is high, the distance for speed adjustment is mainly influenced by the interactions between vehicles, as indicated by the headway. Thus, the term $\triangle x$ is expressed mathematically as a hybrid sigmoid function of the individual headway and acceleration, as follows:

$$
\begin{gathered}
S_{h}(y) \cdot h_{i}+S_{a c c}(y) \cdot F(a c c) \\
S_{h}(y)=\frac{1}{1+e^{-y}} \\
S_{a c c}(y)=\frac{e^{-y}}{1+e^{-y}} \\
y=\tan \left[\left(\frac{\rho_{i}(k)}{\rho_{0}}-\rho^{\wedge}\right) \pi\right] \\
F(a c c)=\frac{v_{i+1}^{2}(k)-v_{i}^{2}(k)}{2 \times a c c_{i}(k)}
\end{gathered}
$$

where $h_{i}=$ averaged individual headway of vehicles in segment $i(\mathrm{~km}), F(a c c)=$ distance function of averaged 


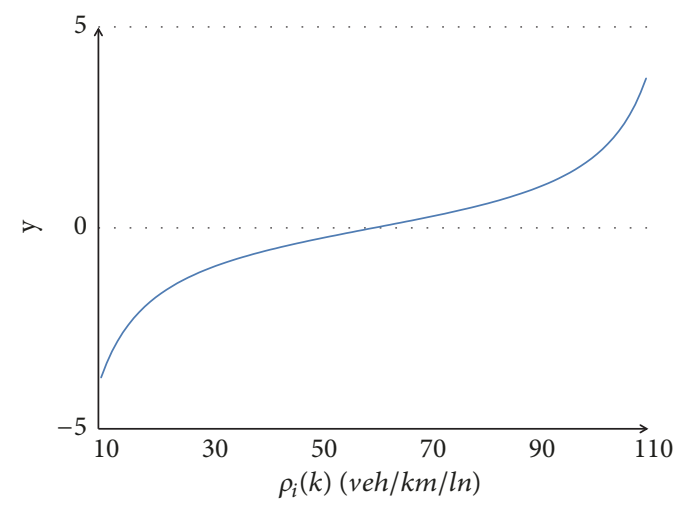

(a)

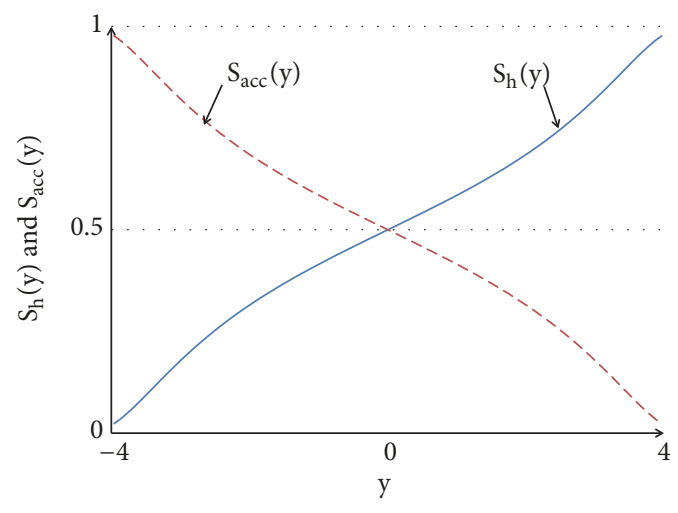

(b)

FIGURE 2: Interpretation of the term $\triangle x$ : (a) the formulated sensitivity y along density $\rho_{i}(k)$ and (b) RELATIONSHIPS between $S_{h}(y), S_{a c c}(y)$, and $y$.

individual acceleration of vehicles $(\mathrm{km}), S_{h}(y), S_{a c c}(y)=$ coefficient related to density, $\rho_{i}(k)=$ density of segment $i$ at time $k(\mathrm{veh} / \mathrm{km} / \mathrm{ln}), \rho^{\wedge}, \rho_{0}=$ model parameter for shaping the sigmoid function, $y=$ function of density, $v_{i}(k)=$ mean speed of segment $i$ at time $k(\mathrm{~km} / \mathrm{h})$, and $\operatorname{acc}_{i}(k)=$ averaged individual acceleration of vehicles of segment $i$ at time $k\left(\mathrm{~km} / \mathrm{h}^{2}\right)$.

The mathematical interpretation of established sigmoid model (13) is illustrated in Figure 2.

As demonstrated in Figure 2(a), in data set the density ranges from 10 to 110 while $y$ ranges approximately from -4 to 4 . The value of $S_{h}(y)$ and $S_{a c c}(y)$ are continuous between 0 and 1. As shown in Figure 2(b), when the density is relatively large, the sensitivity depends mainly on $S_{h}(y)$, as observed in the field. And going the other way around as the density decreases. This means for better representing of the traffic flow dynamics, the formulated sensitivity term was mainly influenced by the individual vehicle at large densities and by the vehicle acceleration status in the low-density region. The formulated sensitivity term is less sensitive when the density is either too large or too low, and otherwise when the density is moderate. Noted that through this modification, the extra constant model parameter $\kappa$ in the basic MEATNET model was neglected since no compensation is needed. The proposed microscopic METANET model improved the prediction accuracy, as shown in Figure 3, and the speed predicted by the modified METANET model is closer to the actual data.

Then, the density and volume of segment $i$ at the next time step are determined using (3) and (4). The speed is determined by the modified model:

$$
\begin{aligned}
v_{i}(k+1)= & v_{i}(k)+\frac{T}{\tau}\left(U\left[\rho_{i}(k)\right]-v_{i}(k)\right) \\
& -\frac{T}{L_{i}} v_{i}(k)\left[v_{i}(k)-v_{i-1}(k)\right]+\frac{\gamma T}{\tau L_{i}} \\
& \cdot\left[\frac{1}{1+e^{-y}} \cdot h_{i}+\frac{e^{-y}}{1+e^{-y}} \cdot F(a c c)\right] \\
& \cdot\left[\rho_{i+1}(k)-\rho_{i}(k)\right]
\end{aligned}
$$

where $y$ is given by (16).
In the MPC-based VSL control strategy proposed in this paper, (19) is derived from the proposed microscopic METANET model. The desired speed $U\left[\rho_{i}(k)\right]$ is replaced by the speed limit $u_{i}(k)$. Thus, the prediction speed under the VSL control is determined by

$$
\begin{aligned}
v_{i}(k+1)= & v_{i}(k)+\frac{T}{\tau}\left(u_{i}(k)-v_{i}(k)\right) \\
& -\frac{T}{L_{i}} v_{i}(k)\left[v_{i}(k)-v_{i-1}(k)\right]+\frac{\gamma T}{\tau L_{i}} \\
& \cdot\left[\frac{1}{1+e^{-y}} \cdot h_{i}+\frac{e^{-y}}{1+e^{-y}} \cdot F(a c c)\right] \\
& \cdot\left[\rho_{i+1}(k)-\rho_{i}(k)\right]
\end{aligned}
$$

3.1.3. Constraints. The constraints of the METANET model by Cao et al. [33] are adopted in this paper. Let $V_{\min }, V_{\max }$ and $V_{d}$ be the minimum speed, maximum speed, and maximum speed difference. Then, based on safety, driver compliance, traffic-flow characteristics, and other considerations, the speed limit is determined based on the following constraints:

(1) To guarantee drivers' safety, the optimal speed limit must be lower than the maximum speed:

$$
u_{i}(k) \leq \mathrm{V}_{\max }
$$

(2) To maintain operating efficiency, the optimal speed limit must be higher than the minimum speed:

$$
u_{i}(k) \geq \mathrm{V}_{\min }
$$

(3) For safe operation, the difference in the speed limits of two consecutive time steps should be less than the maximum difference:

$$
u_{i}(k)-u_{i}(k+1) \leq \mathrm{V}_{\mathrm{d}}
$$

(4) Not all vehicle drivers are able to drive at the speed limit. Therefore, to ensure that the speed limit is 


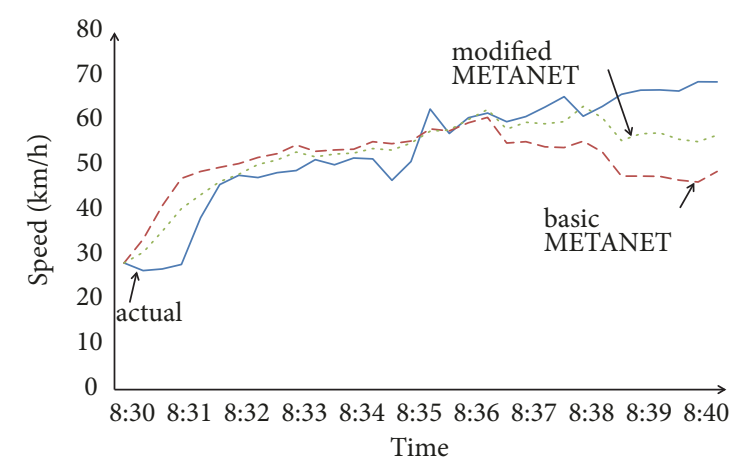

(a)

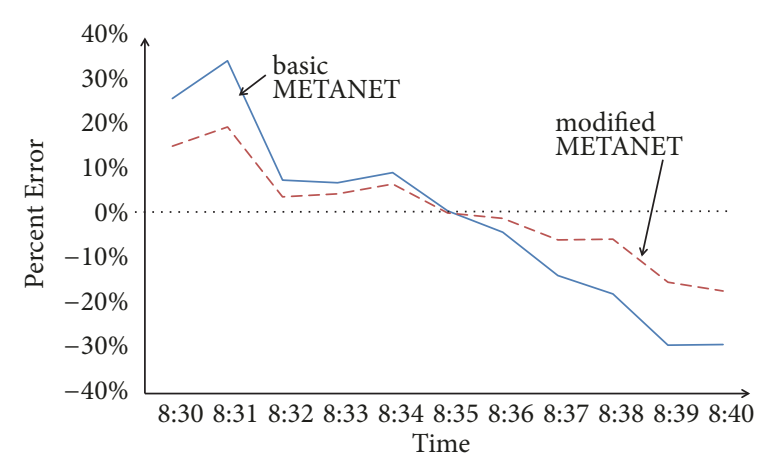

(b)

FIGURE 3: Comparison of speeds of basic and modified METANET models: (a) actual and predicted speeds and (b) percentile prediction error of basic and modified METANET model.

more suitable for actual traffic conditions, the difference between the optimal speed limit and the speed detected downstream should be less than the maximum difference:

$$
\left|u_{i}(k)-v_{i+1}(k-1)\right| \leq \mathrm{V}_{\mathrm{d}}
$$

3.2. Model Validations. To calibrate the proposed model modification, the parameters of the basic and modified METANET (18) and (19) were first calibrated using field data. The data for the modified model were collected on a typical freeway (Whitemud Drive freeway, Edmonton, Canada). The actual data were collected for 100 continuous days. Fifty days of the data were used for model calibration, where model performance was measured using the error between predicted and actual values. The optimal parameters that produced the minimum error were selected.

The other 50 days of data were used for model validation. These data and the optimal parameters were used in the prediction model to predict traffic-flow state. As shown in Figure 3, before 8:35 am, the speed predicted by the modified METANET model is lower than the speed predicted by the basic METANET model, which is closer to the actual speed. The largest percentage of prediction error reduction is $14.9 \%$. After $8: 35 \mathrm{am}$, the speed predicted by the modified METANET model is generally larger than that predicted by the basic METANET model, which is also closer to the actual speed. The largest errors of the basic METANET model for the high and low-density ranges are $34.7 \%$ and $-29.1 \%$, respectively, while those of the modified model are $19.8 \%$ and $-17.0 \%$, respectively. The results show that the modified model has reduced the errors of the basic METANET model for the two density ranges by $14.9 \%$ and $14.1 \%$ in average.

3.3. Model Predictive Control. In this paper, an MPC framework is used to solve the problem of optimal speed limit for implementing proactive VSL control. In MPC, the time horizon is $k$ discrete time steps. As shown is Figure 4, at each time step $k$, the optimal speed limits are computed over a prediction horizon $N_{p}$. The current traffic state variables are used as input to determine the optimal speed limits.
Based on collected traffic data, for every input, the VSLcontrolled future traffic states over the $N_{p}$ horizon are predicted by the prediction model at the current time. The control input implements a control interval $T_{C}$, which is selected to improve traffic conditions.

As shown in Figure 5, the traffic flow in segment $i$ is continuous, namely, it follows the conservation equation of fluid motion. The speed, density, and volume are temporal and spatial variables for each freeway segment. Thus, the three traffic-flow variables are functions of time and position. The variables $\rho_{i}(k)$ and $q_{i}(k)$ represent the density and volume at a certain moment and position, respectively, while $t$ is time and $x$ is coordinate of the position along the driving direction.

The objective function of VSL optimization was set as the weighted sum of total time spent (TTS) and total travel distance (TTD), in order to improve the mobility of the network, as suggested by Cao et al. [33] and couple other previous studies. The speed limits associated with the minimum objective function are selected as the optimal speed limits. The traffic states are updated dynamically after adopting the optimal speed limits, which are then forwarded to the framework again for optimizing the control input in the next time step. The objective function is given by

$$
\begin{aligned}
J= & \sum_{j=1}^{N_{p}} \sum_{i=1}^{M}\left(\alpha_{T T S} T \lambda_{i} L_{i} \rho_{i}(k-1+j)\right. \\
& \left.-\alpha_{T T D} T \lambda_{i} L_{i} \rho_{i}(k-1+j) v_{i}(k-1+j)\right)
\end{aligned}
$$

where $N_{p}=$ total time step, $M=$ number of segments, $T=$ time step of the evolution of traffic flow, $\lambda_{i}=$ number of lanes of segment $i, L_{i}=$ length of segment $i(\mathrm{~km}), \rho_{i}(k-1+j)=$ density of segment $i$ at time $k-1+j(\mathrm{veh} / \mathrm{km} / \mathrm{ln}), v_{i}(k-1+j)=$ mean speed of segment $i$ at time $k-1+j(\mathrm{veh} / \mathrm{km} / \mathrm{ln})$, and $\alpha_{T T S}$ and $\alpha_{\text {TTD }}=$ weighting factors for TTS and TTD, respectively.

\section{Application}

4.1. Simulation Experiment. To evaluate and analyze the MPC-based VSL control strategy using the modified METANET model, an urban freeway corridor is selected as the experimental simulation site. The selected freeway 
TABLE 1: Comparison of the objective function for no VSL, basic and modified METANET-VSL controls.

\begin{tabular}{lccc}
\hline Time & & \multicolumn{2}{c}{ Objective Function } \\
& No VSL & Basic METANET & Modified METANET \\
\hline $6: 30-6: 50$ & 392.3 & 381.1 & 381.1 \\
$6: 50-7: 10$ & 610.0 & 798.6 & 806.5 \\
$7: 10-7: 30$ & 2739.6 & 2539.9 & 2505.5 \\
$7: 30-7: 50$ & 5527.7 & 2932.8 & 3465.9 \\
$7: 50-8: 10$ & 8613.2 & 2589.7 & 2515.2 \\
$8: 10-8: 30$ & 9445.6 & 2617.7 & 1049.4 \\
$8: 30-8: 50$ & 3779.2 & 736.5 & 624.9 \\
\hline Total & 31107.6 & 12596.3 & 11348.5 \\
\hline
\end{tabular}

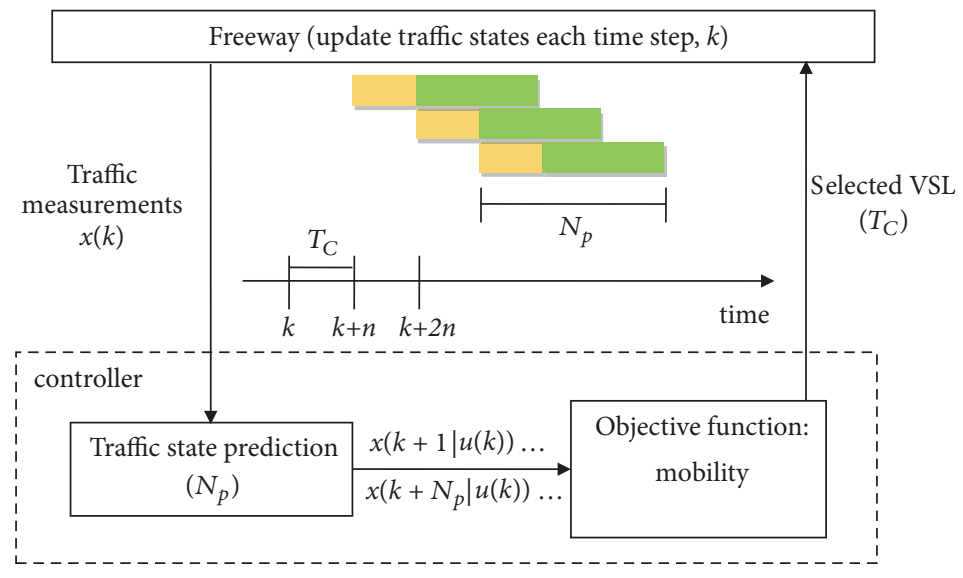

FIGURE 4: MPC-based control framework.

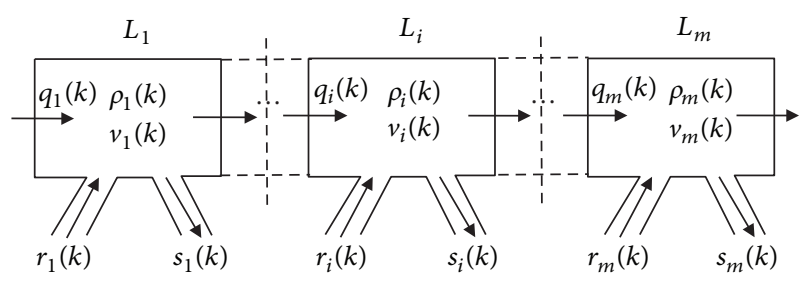

FIgURE 5: Schematic diagram of the METANET model.

is about $9 \mathrm{~km}$ long with three lanes in each direction. For modeling, the corridor is further divided into 13 segments including five on-ramps $\left(r_{1}, r_{2}, r_{3}, r_{4}\right.$, and $\left.r_{5}\right)$ and six offramps $\left(s_{1}, s_{2}, s_{3}, s_{4}, s_{5}\right.$, and $\left.s_{6}\right)$, and the VSL board locations are marked, as shown in Figure 6.

The traffic data were collected on-site using loop detectors installed in each segment, and the experiment was conducted for a peak-hour period of two and a half hour. The VISSIM simulation software was selected to establish the network simulation platform. The authors chose 10 random simulation seeds in the experiment, and the all simulation results in this paper are based on the average of the 10 different scenarios. The simulation resolution is 5 per second in this paper, since a higher resolution will lead to high computational load. Using MATLAB, the MPC-based VSL control strategy that includes the modified METANET model was implemented on the simulated site. The simulation platform was calibrated by minimizing the difference between actual and predicted traffic state variables.

In the experiment, three different control scenarios were evaluated in the simulation platform: (1) no control, (2) VSL control based on the basic METANET model, and (3) VSL control based on the modified METANET model.

4.2. Analysis Results. The basic demand profile of the experiment site is shown in Figure 7 as the volume variations in the uncontrolled scenario. On the profile, two bottlenecks can be recognized around segments $\mathrm{L}_{4}$ and $\mathrm{L}_{8}$. At Segment $\mathrm{L}_{4}$, parts of the vehicles entering the mainline freeway through on-ramp $r_{1}$ want to leave the mainline through off-ramp $s_{2}$. The weaving segment in-between results in chaotic traffic operation and serious congestion. A similar weaving section exists around segment $\mathrm{L}_{8}$, and significant capacity drop can be observed as well.

The evaluation results of the objective function for NoVSL, basic METANET-VSL, and modified METANET-VSL controls (using an interval of $20 \mathrm{~s}$ ) are shown in Table 1 and Figure 8. As illustrated, under free-flow condition (before 7:00 AM), traffic conditions of the three tested scenarios are nearly identical. After 7:10 am, the traffic demand continued to increase. In both METANET-VSL controlled scenarios, 


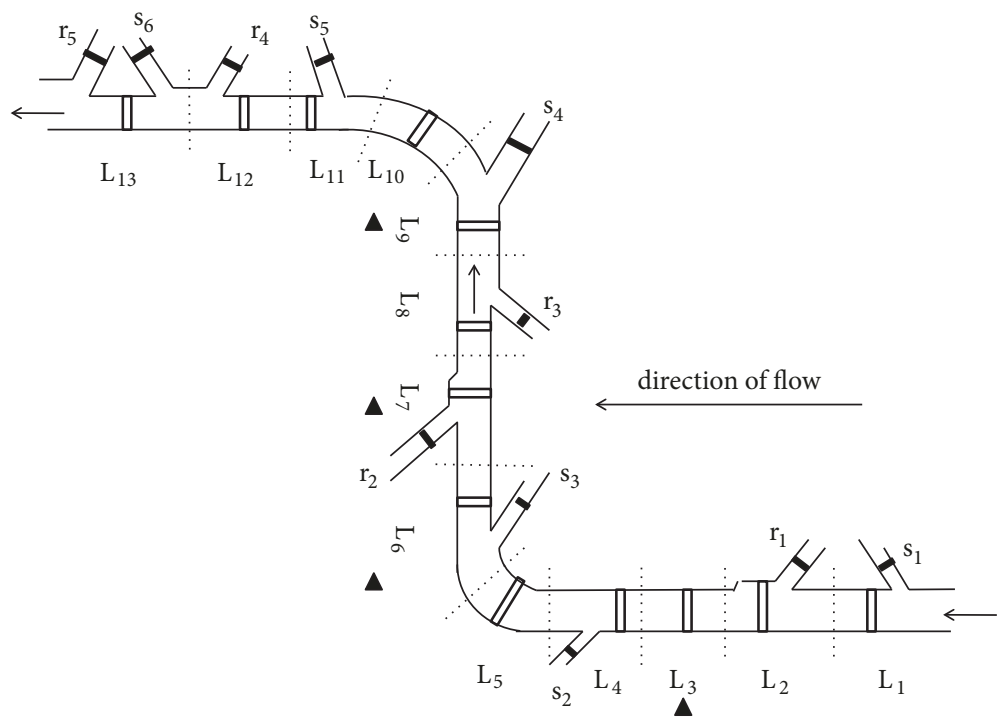

Mainline loop detector location

Ramp loop detector location

$\Delta$ VSL board location

FIGURE 6: Schematic diagram of expressway segments.

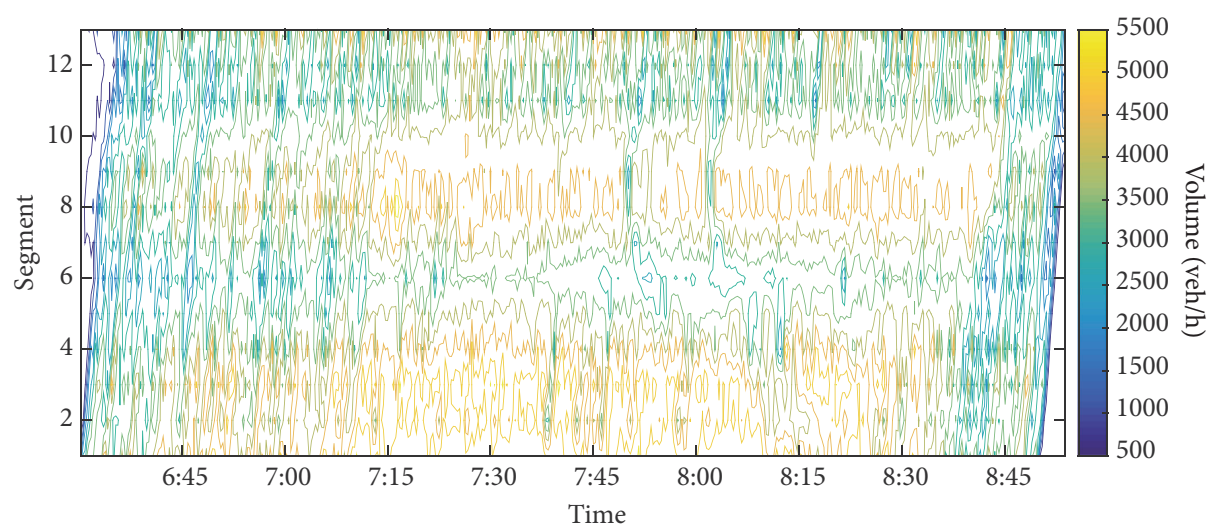

FIGURE 7: Volume variation at different segments without control.

the adopted traffic flow model was able to predict the forthcoming traffic breakdown and determined to advise reduced speed limit in advance for overall speed stability (6:50 to 7:10 AM). Thus, in the time slice, the objective function values in both controlled scenario were worse than the uncontrolled case. In addition, since the proposed model modification captures the speed dynamics more accurately during the density oscillation, the proposed modified control scenario determines to maintain a lower VSL value at 7:30 to $7: 50$ AM. As a result, the objective function performance in that time slice is not as good as the basic METANET controlled scenario, which mistakenly predicted the traffic flow has recovered and raised the VSL. Instead, the proposed modified VSL control maintained a more stable and sustainable traffic flow, which avoids the traffic breakdown occurred later in the basic METANET controlled scenario shortly after 8:00 AM. Overall speaking, the combined TTS and TTD for the basic and proposed modified METANET controlled scenario were reported to be $12,596.3$ and 11,348.5, indicating an improvement of $10 \%$ in terms of the mobility performance and even more significant when comparing with the uncontrolled scenario. Clearly, the modified METANET-based VSL control strategy plays a better role in improving traffic mobility.

For segment densities, a comparison of no-VSL and basic and modified METANET-VSL controls is shown in Figure 9. The density of Segment $\mathrm{L}_{8}$ increased significantly since around 7:30 am and remained high until nearly the end of the study period. The congestion propagates upstream to $\mathrm{L}_{7}$ and such. It can be observed that both the severity and duration of the congestion has been significantly reduced 


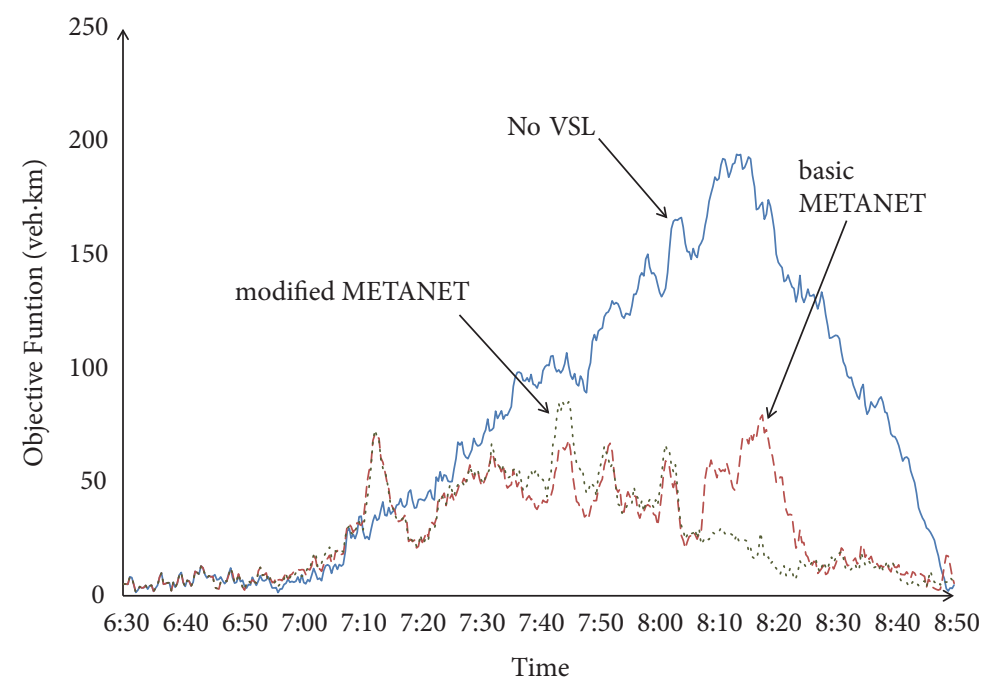

FIGURE 8: Comparison of the objective function for No VSL, basic, and modified METANET-VSL controls.

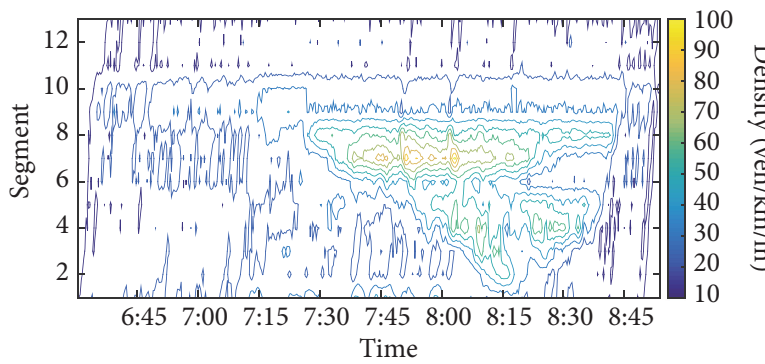

(a)

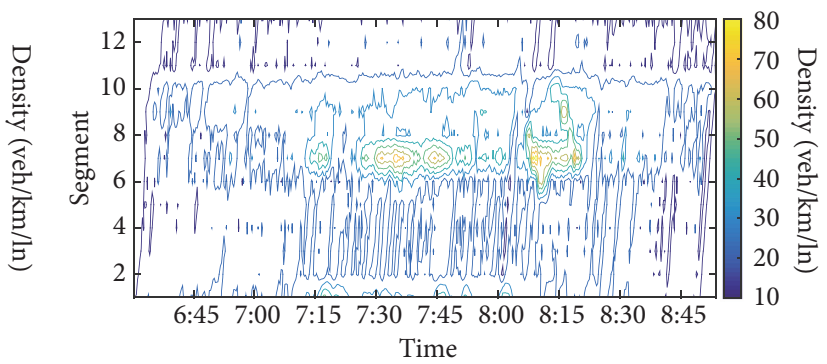

(b)

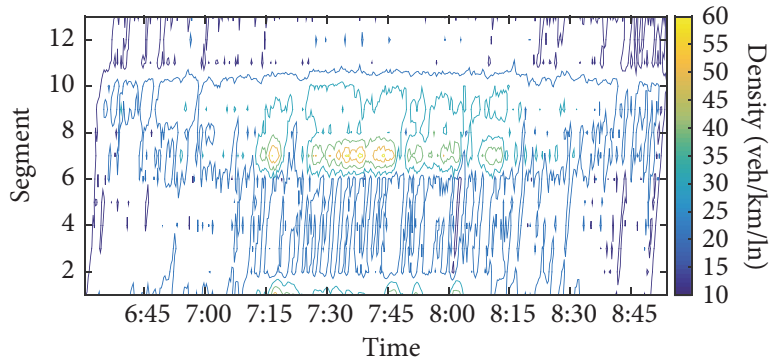

(c)

FIGURE 9: Comparison of segment densities of No-VSL, basic, and modified METANET-VSL controls: (a) no-VSL control; (b) basic METANET model; (c) modified METANET model.

by the deployed VSL control strategy, especially in the two identified bottlenecks (segment $\mathrm{L}_{4}$ and $\mathrm{L}_{8}$ ). From 8:00 to 8:15 am, the density of segment $\mathrm{L}_{8}$ has been maintained at approximately $70 \mathrm{veh} / \mathrm{km} / \mathrm{ln}$ for basic METANET model control and around $40 \mathrm{veh} / \mathrm{km} / \mathrm{ln}$ for proposed modified METANET VSL control.

By capturing the variations of the speed dynamics, the METANET-model activated the VSL to prevent capacity drop and relieve traffic congestion. Taking Segment $\mathrm{L}_{8}$ as an example, under modified-METANET control, comparison between the speed limit and the actual speed was shown in Figure 10. Before 6:50 am, the modified-METANET VSL control was not activated since the traffic congestion did not emerge before 7:00 am. Before that congestion, the modified-METANET model had predicted the speed drop and lowered the speed limit in advance. As a result, the modified-METANET model improved the minimum segment speed to approximately $40 \mathrm{~km} / \mathrm{h}$ and shortened the congestion duration. After the congestion relieved, the speed limit recovered gradually.

\section{Conclusions}

This paper proposed a modified METANET model that utilizes the microscopic traffic-flow data. A MPC frameworkbased control strategy incorporates the proposed modified 


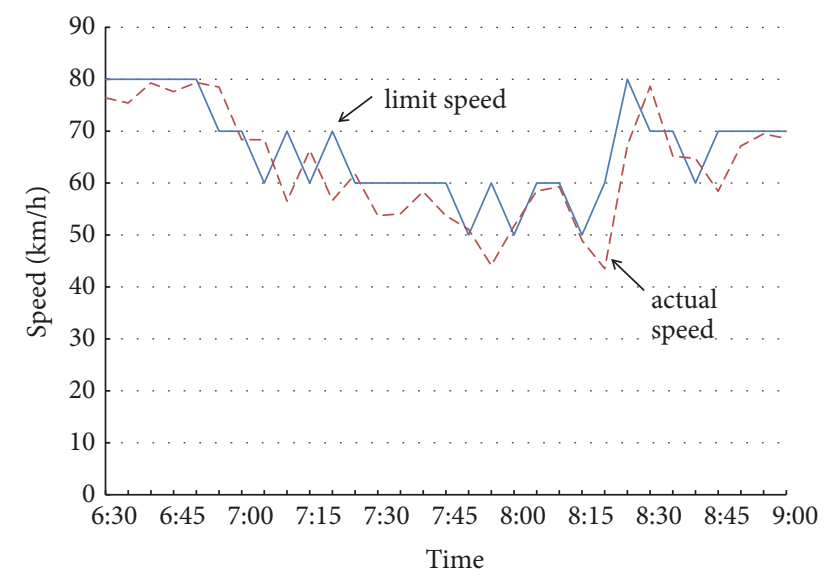

FIGURE 10: Comparison between limit speed and actual speed under modified METANET control.

model was established to capture the variations of traffic flow dynamics, which enables the VSL control to prevent dramatic decline of link speed beforehand and gains improvement on the freeway mobility performance.

The proposed formulation of the anticipation term in the modified METANET model is more reasonable and comprehensive. The proposed modification takes in the microscopic traffic-flow data, such as individual vehicle headway and accelerations, to better interpreted the progress of drivers adjusting toward the anticipated speed. The proposed modified model can produce more accurate prediction results, which provides a more reliable basis for achieving further improvement in VSL control applications. The modified METANET model has reduced the prediction error by up to $14.9 \%$ and $14.1 \%$ for the low and high-density ranges, respectively.

As a result, traffic mobility was substantially improved along with the reduced prediction error. The VISSIM software was used to establish a field data based experimental simulation platform for model validations. To evaluate the control benefits, the traffic-flow states of the modified METANET-based VSL control strategy were compared with those of the No-VSL control and basic METANET-based VSL controlled scenario. The modified METANET model has achieved substantial improvements in terms of mobility performance. The simulation results demonstrated that the modified METANET model reduced segment density, increased segment speed, and shortened the congestion period, indicates the improved freeway mobility. More initiatives to aid the speed prediction of the model should continue to be explored in the future.

\section{Data Availability}

The data used to support the findings of this study may be released upon application to the authors, who can be contacted at fangjie@fzu.edu.cn.

\section{Conflicts of Interest}

The authors declare that they have no conflicts of interest.

\section{References}

[1] H. Dai, E. Yao, N. Lu et al., "Freeway network connective reliability analysis based complex network approach," Procedia Engineering, vol. 137, pp. 372-381, 2016.

[2] N. Cui, B. Chen, K. Zhang, Y. Zhang, X. Liu, and J. Zhou, "Effects of route guidance strategies on traffic emissions in intelligent transportation systems," Physica A: Statistical Mechanics and its Applications, vol. 513, pp. 32-44, 2019.

[3] Y. E. Hawas, G. Thandavarayan, B. Basheerudeen, and M. Sherif, "Testbed evaluation of real-time route guidance in intervehicular communication urban networks," IEEE Access, vol. 7, pp. 1470-1485, 2019.

[4] A. Janota, P. Holečko, M. Gregor, and M. Hruboš, "Rampmetering algorithms evaluated within simplified conditions," Journal of Civil \& Environmental Engineering, vol. 13, no. 2, pp. 112-119, 2017.

[5] Y. Li, N. Duan, and X. Yang, "Analysis balance parameter of optimal ramp metering," IOP Conference Series: Materials Science and Engineering, vol. 359, no. 1, article 012007, 2018.

[6] M. Papageorgiou, E. Kosmatopoulos, and I. Papamichail, "Effects of variable speed limits on motorway traffic flow," Transportation Research Record, no. 2047, pp. 37-48, 2008.

[7] R. Jiang, A. B. Lucky, and E. Chung, "Calibration and operational analysis of variable speed limits for high flow conditions," in Proceedings of the IEEE Forum on Integrated and Sustainable Transportation Systems, FISTS '11, pp. 221-226, 2011.

[8] M. Islam, M. Hadiuzzaman, J. Fang, T. Qiu, and K. El-Basyouny, "Assessing mobility and safety impacts of a variable speed limit control strategy," Transportation Research Record, vol. 2364, pp. $1-11,2013$.

[9] Z. Xu, T. Wei, S. Easa, X. Zhao, and X. Qu, "Modeling relationship between truck fuel consumption and driving behavior using data from internet of vehicles," Computer-Aided Civil and Infrastructure Engineering, vol. 33, no. 3, pp. 209-219, 2018.

[10] M. Papageorgiou, J.-M. Blosseville, and H. Hadj-Salem, "Modelling and real-time control of traffic flow on the southern part of boulevard peripherique in Paris: Part I: modelling," Transportation Research Part A: General, vol. 24, no. 5, pp. 345359, 1990.

[11] C. Lee, B. Hellinga, and F. Saccomanno, "Real-time crash prediction model for application to crash prevention in freeway traffic," Transportation Research Record: Journal of the Transportation Research Board, no. 1840, pp. 67-77, 2003.

[12] C. Lee, B. Hellinga, and F. Saccomanno, "Evaluation of variable speed limits to improve traffic safety," Transportation Research Part C: Emerging Technologies, vol. 14, no. 3, pp. 213-228, 2006.

[13] R. C. Carlson, I. Papamichail, and M. Papageorgiou, "Local feedback-based mainstream traffic flow control on motorways using variable speed limits," IEEE Transactions on Intelligent Transportation Systems, vol. 12, no. 4, pp. 1261-1276, 2011.

[14] R. C. Carlson, I. Papamichail, and M. Papageorgiou, "Comparison of local feedback controllers for the mainstream traffic flow on freeways using variable speed limits," Journal of Intelligent Transportation Systems: Technology, Planning, and Operations, vol. 17, no. 4, pp. 268-281, 2013.

[15] G.-R. Iordanidou, I. Papamichail, C. Roncoli, and M. Papageorgiou, "A feedback-based approach for mainstream traffic flow control of multiple bottlenecks on motorways," IFAC Proceedings Volumes, vol. 47, no. 3, pp. 11344-11349, 2014.

[16] G.-R. Iordanidou, C. Roncoli, I. Papamichail, and M. Papageorgiou, "Feedback-based mainstream traffic flow control for 
multiple bottlenecks on motorways," IEEE Transactions on Intelligent Transportation Systems, vol. 16, no. 2, pp. 610-621, 2015.

[17] M. Burger, M. Van Den Berg, A. Hegyi, B. De Schutter, and J. Hellendoorn, "Considerations for model-based traffic control," Transportation Research Part C: Emerging Technologies, vol. 35, pp. 1-19, 2013.

[18] B. Khondaker and L. Kattan, "Variable speed limit: a microscopic analysis in a connected vehicle environment," Transportation Research Part C: Emerging Technologies, vol. 58, pp. 146-159, 2015.

[19] J. Zhang, A. Boitor, and P. Ioannou, "Design and evaluation of a roadway controller for freeway traffic," in Proceedings of the 8th International IEEE Conference on Intelligent Transportation Systems '05, pp. 543-548, IEEE, 2005.

[20] S. K. Zegeye, B. De Schutter, H. Hellendoorn, and E. Breunesse, "Reduction of travel times and traffic emissions using model predictive control," in Proceedings of the 2009 American Control Conference, ACC '09, pp. 5392-5397, IEEE, 2009.

[21] A. H. Ghods, L. Fu, and A. Rahimi-Kian, "An efficient optimization approach to real-time coordinated and integrated freeway traffic control," IEEE Transactions on Intelligent Transportation Systems, vol. 11, no. 4, pp. 873-884, 2010.

[22] C. F. Daganzo, “The cell transmission model: a dynamic representation of highway traffic consistent with the hydrodynamic theory," Transportation Research Part B: Methodological, vol. 28, no. 4, pp. 269-287, 1994.

[23] M. Hadiuzzaman, T. Z. Qiu, and X.-Y. Lu, "Variable speed limit control design for relieving congestion caused by active bottlenecks," Journal of Transportation Engineering, vol. 139, no. 4, pp. 358-370, 2012.

[24] M. Hadiuzzaman and T. Z. Qiu, "Cell transmission model based variable speed limit control for freeways," Canadian Journal of Civil Engineering, vol. 40, no. 1, pp. 46-56, 2013.

[25] Y. Han, A. Hegyi, Y. Yuan, S. Hoogendoorn, M. Papageorgiou, and C. Roncoli, "Resolving freeway jam waves by discrete firstorder model-based predictive control of variable speed limits," Transportation Research Part C: Emerging Technologies, vol. 77, pp. 405-420, 2017.

[26] A. Muralidharan and R. Horowitz, "Computationally efficient model predictive control of freeway networks," Transportation Research Part C: Emerging Technologies, vol. 58, pp. 532-553, 2015.

[27] A. Hegyi, Model Predictive Control for Integrating Traffic Control Measures, TRAIL Research School, Netherlands, 2004.

[28] A. Hegyi, B. de Schutter, and J. Hellendoorn, "Optimal coordination of variable speed limits to suppress shock waves," IEEE Transactions on Intelligent Transportation Systems, vol. 6, no. 1, pp. 102-112, 2005.

[29] A. Hegyi, B. De Schutter, and H. Hellendoorn, "Model predictive control for optimal coordination of ramp metering and variable speed limits," Transportation Research Part C: Emerging Technologies, vol. 13, no. 3, pp. 185-209, 2005.

[30] R. C. Carlson, I. Papamichail, M. Papageorgiou, and A. Messmer, "Optimal motorway traffic flow control involving variable speed limits and ramp metering," Transportation Science, vol. 44, no. 2, pp. 238-253, 2010.

[31] R. Sun, J. Hu, X. Xie, and Z. Zhang, "Variable speed limit design to relieve traffic congestion based on cooperative vehicle infrastructure system," Procedia: Social and Behavioral Sciences, vol. 138, pp. 427-438, 2014.
[32] R. Yu and M. Abdel-Aty, "An optimal variable speed limits system to ameliorate traffic safety risk," Transportation Research Part C: Emerging Technologies, vol. 46, pp. 235-246, 2014.

[33] J. Cao, D. Hu, Y. Luo, T. Z. Qiu, and Z. Ma, "Exploring the impact of a coordinated variable speed limit control on congestion distribution in freeway," Journal of Traffic and Transportation Engineering, vol. 2, no. 3, pp. 167-178, 2015. 


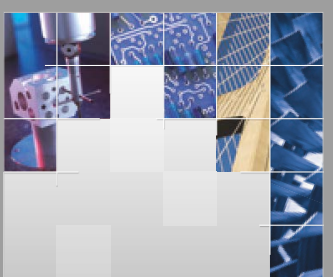

\section{Enfincering}
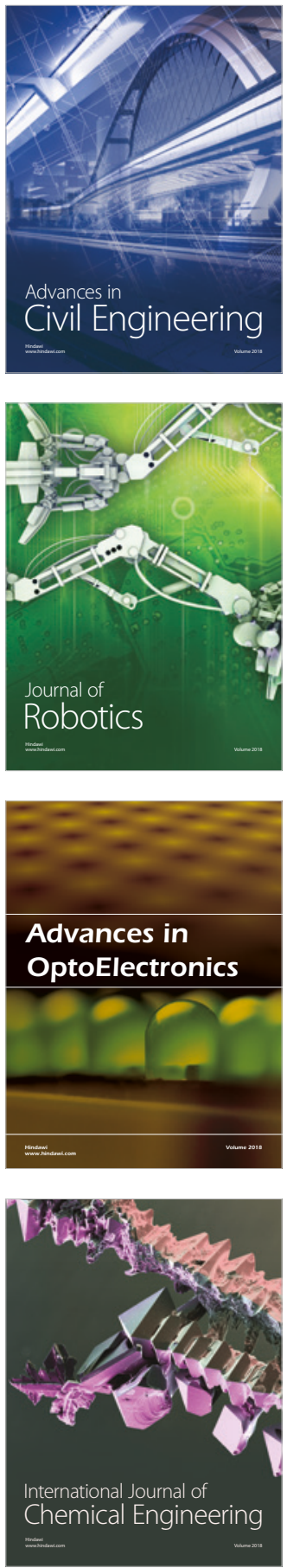

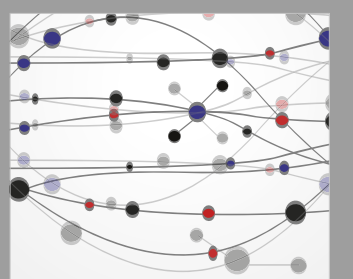

\section{Rotating \\ Machinery}

The Scientific World Journal

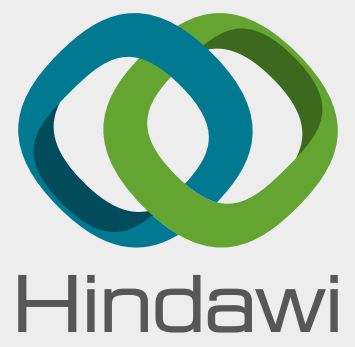

Submit your manuscripts at

www.hindawi.com
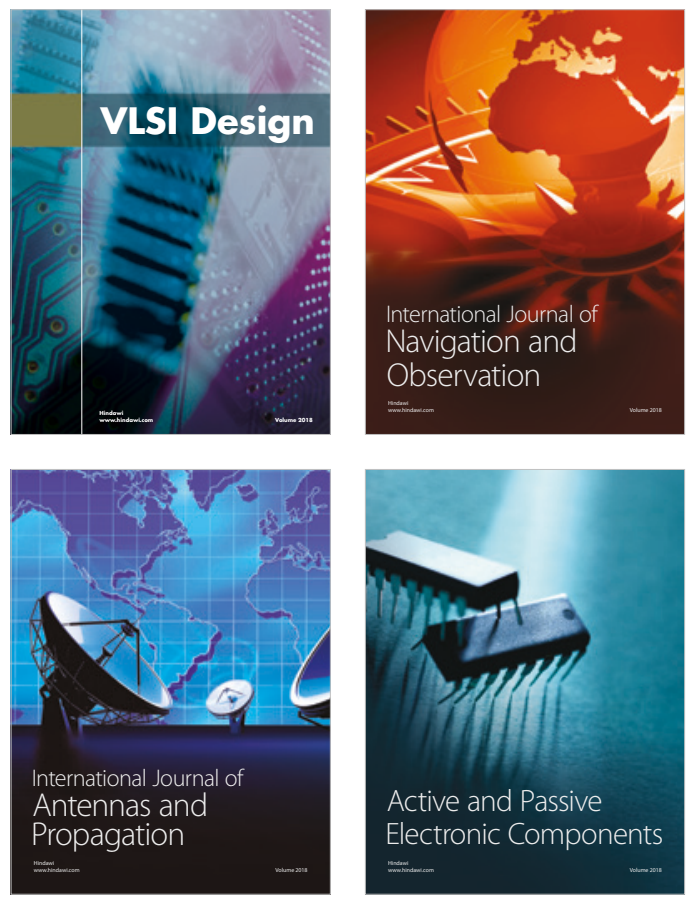
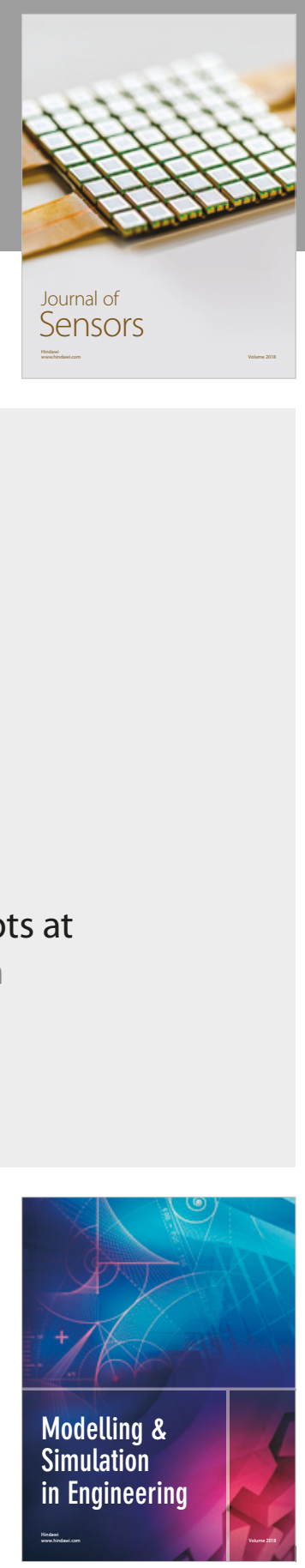

\section{Advances \\ Multimedia}
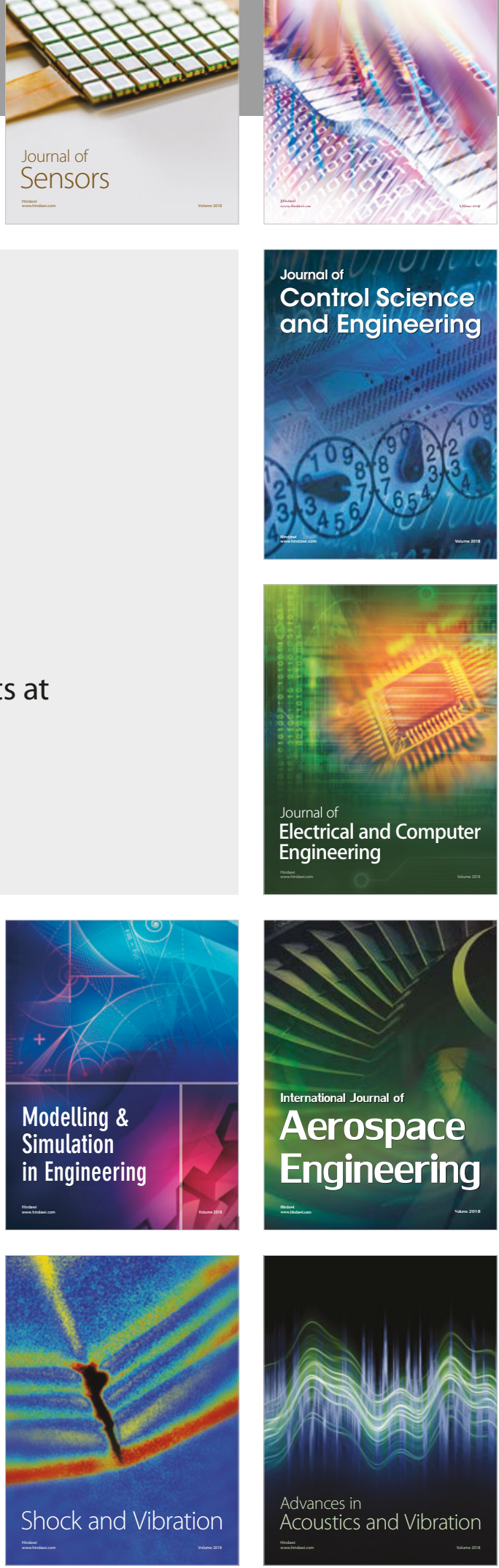